\title{
THE PERFORMANCE ANALYSIS OF OFDM SYSTEMS WITH USING DIFFERENT ORDER OF QAM
}

\author{
Zainab Mones, \\ Zainab_mones81@yahoo.com \\ Ahmed El.barcha \\ Electrical Engineering Department, Faculty of Engineering, \\ University of Garyounis Libya
}

\begin{abstract}
Orthogonal frequency division multiplexing (OFDM) is a special case of multicarrier transmission, where a single high rate datastream is transmitted over a number of lower rate datastream. The demand for high-speed mobile wireless communications is rapidly growing. OFDM technology is a key technique for achieving the high data capacity and spectral efficiency requirements for wireless communication systems nowadays. In this paper, we investigate the performance of OFDM systems with different orders of Quadrature Amplitude Modulation (QAM). We consider OFDM scheme with an Additive White Gaussian Noise channel (AWGN) and analyze their performance in terms of the bit error rate (BER) in the system.

عبارة عن نوع خاص من الإرسال المتعدد الناقلات حيث إن الإشارة تقسم إلى عدة إشار ات وترسل على

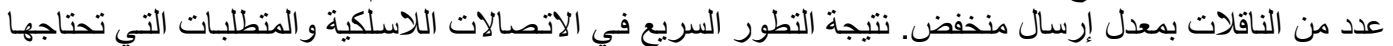

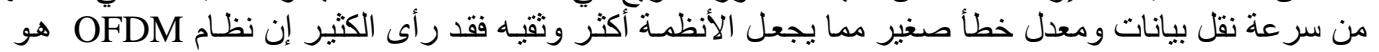

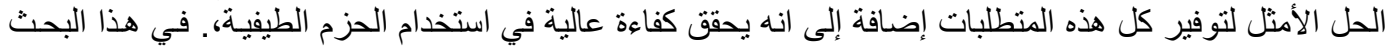

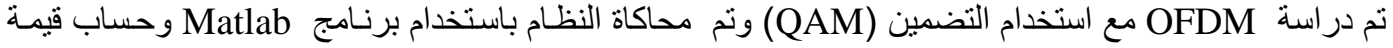

.BER
\end{abstract}

Keywords: OFDM, QAM, BER and spectral efficiency

\section{INTRODUCTION}

OFDM is a multi-carrier transmission technique that has been recently recognized as an excellent method for high speed bi-directional wireless data communication. Its history dates back to the 1960s, but it has recently become popular because economical integrated circuits that can perform the necessary high speed digital operations have become available. OFDM effectively squeezes multiple modulated carriers tightly together, reducing the required bandwidth but keeping the modulated signals orthogonal so they do not interfere with each other. In 1971, Weinstein and Ebert proposed a modified OFDM system in which the DFT was applied to generate the orthogonal subcarriers waveforms [1], [2]. OFDM is similar to FDM but much more spectrally efficient by spacing the subchannels much closer together (until they are actually overlapping). This is done by finding frequencies that are orthogonal, which means that they are perpendicular in a mathematical sense, allowing the spectrum of each subchannel to overlap another without interfering with each other [3], [4], [5].

This paper is organized as follows: the system simulation model is described in section 2. Section 3 presents the results obtained and discusses the performance of the system in term of BER. Finally, conclusions will be drawn in section 4 .

\section{OFDM SYSTEM MODEL}

The models of the system transmitter and receiver are illustrated in Fig. 1. The choice of various OFDM parameters is a trade off between various, often conflicting, requirements. In OFDM design, a number of parameters need to be considered which are number of data sub-carriers, total number of subcarriers, sub-carrier spacing, guard time, symbol duration, sampling rate, modulation type per subcarrier, and FFT length [4]. In the OFDM at the transmitter side, an incoming bit stream, most likely with a high bit rate enters a modulation part to be mapped in PSK (BPSK, QPSK), QAM (16QAM or 64QAM) or other modulations. The incoming data symbols then enter a serial to parallel converter, mapping the high data stream into $\mathrm{N}$ lower-rate (parallel) data streams [6].

If $T$ denotes the symbol interval, the symbol interval in the OFDM system is increased to $N T$, which makes the system more robust against the delay spread [7]. Each data stream then placed on its own carrier and carrier spacing is carefully selected to ensure orthogonality i.e. to ensure that carrier can be separable one from another at the receiver side. Next, the carriers are added together [6]. One trick that makes low cost at the transmitter is the use of an IFFT. Then, guard interval (cyclic prefix) is added by copying the last part of the signal to the first part to simplify ISI mitigation [8], [9]. 


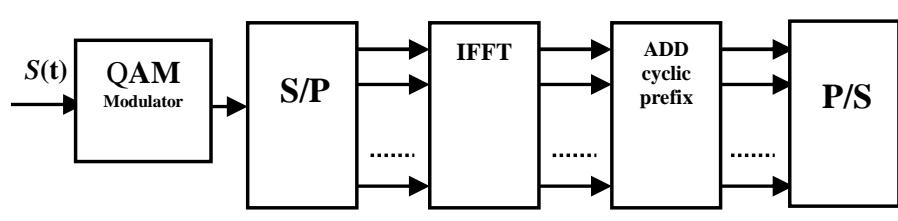

Transmitter

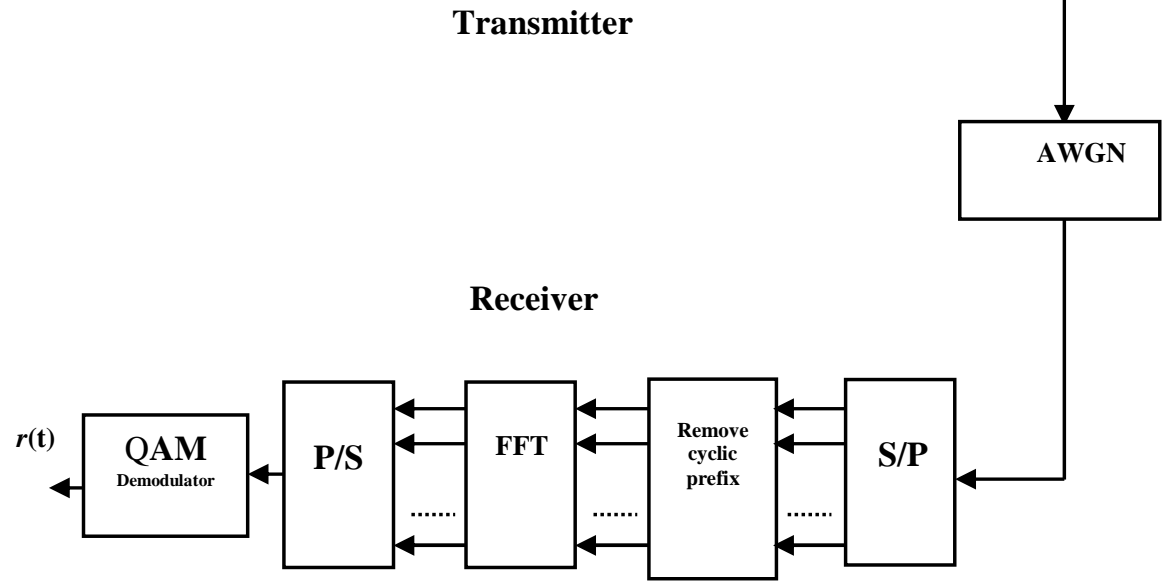

Fig. 1 Block diagrams of the OFDM simulation model

Finally, this signal, called OFDM symbol, is modulated up to the transmit frequency and then sent out across to the channel. The OFDM signal in the complex baseband signalling can be written as:

$$
s(t)=\sum_{k=0}^{N-1} s_{k} e^{j 2 \pi f_{k} t}
$$

If s(t) is sampled at an interval of $T_{s a}=\frac{T_{s}}{N}$, then:

$$
\begin{aligned}
& S_{n}=s\left(n \Delta_{s}\right)=\sum_{k=0}^{N-1} s_{k} e^{j 2 \pi f_{k} \frac{n T_{s}}{N}} \\
& f_{k}=f_{0}+k \Delta f
\end{aligned}
$$

Without loss of generality, setting $f_{0}=0$, then $f_{k} T_{s}=k$ and Eq.(2) becomes:

$$
S_{n}=\sum_{k=0}^{N-1} S_{k} e^{j \frac{2 \pi k n}{N}}=I D F T\left\{S_{k}\right\}
$$

Where IDFT denotes the inverse discrete Fourier transform. Therefore, the OFDM transmitter can be implemented using the IDFT. For the same reason, the receiver can be also implemented using DFT.

$$
S_{k}=\sum_{k=0}^{N-1} s_{n} e^{-j \frac{2 \pi k n}{N}}=D F T\left\{S_{n}\right\} .
$$

In practice, OFDM systems are implemented using a combination of FFT and IFFT blocks that are mathematically equivalent versions of the DFT and IDFT, respectively, because FFT algorithm provides an efficient way to implement the DFT and the IDFT. It reduces the number of complex multiplications from $N^{2}$ to $\frac{N}{2} \log _{2} N$ for an Npoint DFT or IDFT. Hence, with the help of the FFT algorithm, the implementation of OFDM is very simple [10].

\subsection{Bandwidth Efficiency}

For an OFDM signal consisting of $\mathrm{N}$ subchannels, the signal bandwidth is about $(\mathrm{N}+1) \Delta_{f}$. Since the transmission rate of each subchannel is $1 / \mathrm{T}$ symbols/sec., the total transmission rate of OFDM signal is N/T symbol/sec. Therefore, the bandwidth efficiency of the OFDM system in symbols/sec/Hz is

$$
\begin{aligned}
\eta & =\frac{N / T}{(N+1) \Delta_{f}} \\
& =\frac{N /\left(T_{s}+T_{g}\right)}{(N+1) / T_{s}} \\
& =\frac{1}{1+\frac{1}{N}} \frac{1}{1+\frac{T_{g}}{T_{s}}}
\end{aligned}
$$

For most practical OFDM systems, $\mathrm{N}$ is much larger than 1 and the guard interval is much smaller than the OFDM symbol duration, so $\eta \approx 1$. If each symbol carries $\mathrm{k}$ bits information, the bandwidth efficiency will be $\mathrm{k}$ bits/sec/Hz [10]. So, we can define the bandwidth efficiency as the number of bits per second that can be transmitted in one Hertz of system bandwidth [11]. 


\subsection{Assumptions}

In this paper, the system will be assumed ideal, and some assumptions will be as following:

- A transmitter and a receiver are fixed, so that Doppler Effect does not exist.

- Transmitter has only one antenna.

- Receiver has only one antenna.

\section{SIMULATION RESULTS}

In this section calculation of the BER of an OFDM system by using computer simulation program has been made. An OFDM system with $\mathrm{N}$ sub-carriers is represented in Fig. 1. The simulation includes all the stages for transmitter, channel and receiver. Because of the MATLAB sampling time, the transmission was implemented in baseband to avoid long periods of simulation. Figure 2 presents simulation results (BER vs. SNR) of OFDM with MQAM (in different orders) system over AWGN channel. From this figure, SNR which is required in proving target bit error rate can be found as shown in Table 1.

Table 1 Required SNR to maintain a BER below a given threshold

\begin{tabular}{|c|c|c|c|}
\hline Type of $\begin{array}{l}\text { Spectral } \\
\text { modulation }\end{array}$ & \multicolumn{2}{|c|}{ Required SNR (dB) } \\
\cline { 3 - 4 } & Efficiency & BER $<1 \times 10^{-2}$ & BER $<1 \times 10^{-3}$ \\
\hline 4-QAM & 2 & 4.8 & 7.3 \\
\hline 16-QAM & 4 & 8.2 & 10.85 \\
\hline 64-QAM & 6 & 14.3 & 16.7 \\
\hline
\end{tabular}

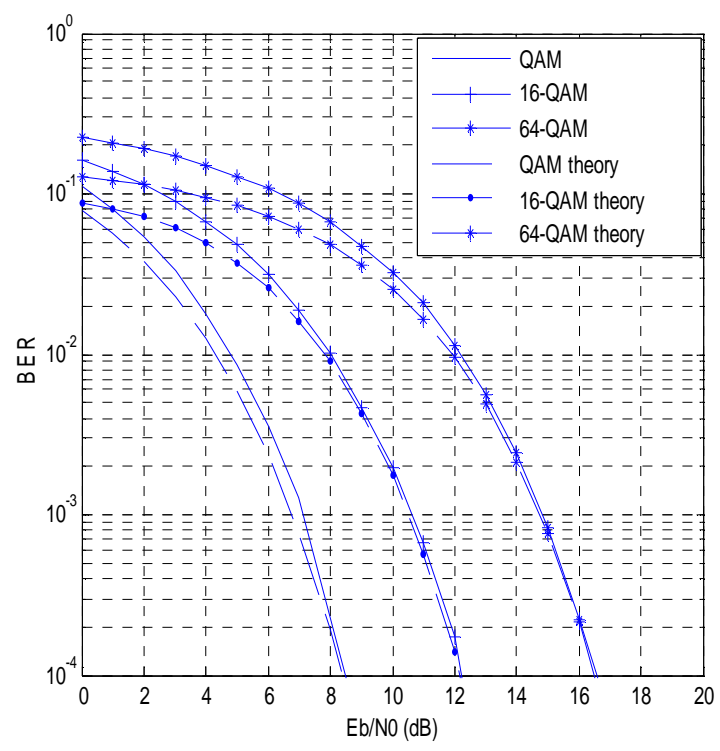

Fig. 2 The BER vs. SNR of OFDM system with QAM modulation over AWGN channel

\section{CONCLUSIONS}

In this paper, the performance of OFDM with different order of QAM was measured in terms of both spectral efficiency and bit error rate. According to the simulation results, OFDM appears to be a good modulation technique for high performance wireless telecommunication. While fixed schemes achieve either good spectral efficiency or good energy efficiency, but not both, so we may use adaptive modulation to increase the performance of OFDM systems.

\section{ACKNOWLEDGEMENT}

We would like to thank our families for their love and continuous support during our life.

\section{REFERENCES}

[1] Marco Tirado, "Analysis, Simulation and Implementation of a SIMO OFDM Communications System", 2006.

[2] Dušan Matiæ,'OFDM as a possible modulation technique for multimedia applications in the range of mm waves", II edition 10/30/98/TUDTVS.

[3] Louis Litwin and Michael Pugel, "The principles of OFDM", 2001, www.rfdesign.com

[4] Rakesh Rajbanshi and Anupama Veeragandham, "OFDM systems Design", 2004.

[5] Dr.S.S.Riaz Ahamed, "Performance Analysis of OFDM", 2008 JATIT.

[6] Hand Book, Edited by Mohamed Ibnkahla," Signal Processing for Mobile Communication", CRC PRESS, 2005.

[7] Tiejun (Ronald) Wang, John G. Proakis, James R. Zeidler "Performance Analysis of High QAM OFDM System Over Frequency Selective Time-Varying Fading Channel " 2003 IEEE. Vol. pp.793-798.

[8] Yasamin Mostofi and Donald C. Cox, "Mathematical Analysis of the Impact of Time Synchronization Error on the Performance of an OFDM System", IEEE comm. 2006, Vol. 54, No.2, pp. 226-230.

[9] Hiroshi Harada and Ramjee Prasad, "Simulation and Software Radio for Mobile Communications".

[10] Ye Geoffrey, "Orthogonal Frequency Division Multiplexing for Wireless Communications", Springer, 2006.

[11]Fuqin Xiong, "Digital Modulation Techniques", Artech House telecommunications library, 2000. 